\title{
Identification of Gai3 as a promising target for osteosarcoma treatment
}

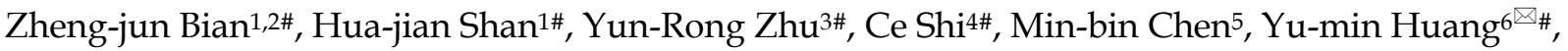 \\ Xiao-dong Wang ${ }^{7 凶}$, Xiao-zhong Zhou ${ }^{1 凶}$ and Cong Cao ${ }^{1,8,9 凶}$ \\ 1. Department of Orthopedics, Clinical Research Center of Neurological Disease, The Second Affiliated Hospital of Soochow University, Suzhou, China. \\ 2. Department of Orthopedics, The Affiliated Hospital of Yangzhou University, Yangzhou University, Yangzhou, China. \\ 3. Department of Orthopedics, Affiliated Jiangyin Hospital of Xuzhou Medical University, Jiangyin, China. \\ 4. Department of Orthopedics, The Affiliated Suqian Hospital of Xuzhou Medical University, Suqian, China. \\ 5. Department of Radiotherapy and Oncology, Kunshan First People's Hospital Affiliated to Jiangsu University, Kunshan, China \\ 6. Department of Orthopedics, The First Affiliated Hospital of Nanjing Medical University, Nanjing, China. \\ 7. Department of Orthopedics, Children's Hospital of Soochow University, Suzhou, China. \\ 8. Jiangsu Key Laboratory of Neuropsychiatric Diseases and Institute of Neuroscience, Soochow University, Suzhou, China. \\ 9. North District, The Affiliated Suzhou Hospital of Nanjing Medical University, Suzhou Municipal Hospital, Suzhou, China. \\ \# Co-first authors.
}

$\triangle$ Corresponding authors: Dr. Yu-min Huang, Department of Orthopedics The First Affiliated Hospital of Nanjing Medical University, Nanjing, 210029, China. Email: xiaoan19860820@126.com; Prof. Xiao-dong Wang, Department of Orthopedics, Children's Hospital of Soochow University, Suzhou, China, 215100. E-mail: xiaodongwangsz@163.com. Prof. Xiao-zhong Zhou, Department of Orthopedics, The Second Affiliated Hospital of Soochow University, San-xiang Rd, Suzhou, China, 215003. Email: zhouxz@suda.edu.cn. Prof. Cong Cao, Clinical Research Center of Neurological Disease, The Second Affiliated Hospital of Soochow University, 199 Ren-ai Road, Suzhou, Jiangsu 215123, China. Tel/Fax: +86-0512-65883658. E-mail: caocong@suda.edu.cn.

(C) The author(s). This is an open access article distributed under the terms of the Creative Commons Attribution License (https://creativecommons.org/licenses/by/4.0/). See http://ivyspring.com/terms for full terms and conditions.

Received: 2021.11.11; Accepted: 2021.12.31; Published: 2022.01.24

\begin{abstract}
Sustained activation of multiple receptor tyrosine kinases (RTKs) simultaneously is vital for tumorigenesis and progression of osteosarcoma (OS). Gai proteins recruitment to various RTKs mediates downstream oncogenic signaling activation. The expression, functions and underlying mechanisms of Gai3 in human OS were examined. Expression of Gai3 is robustly elevated in human OS tissues and is correlated with a poor overall survival. In patient-derived primary OS cells and immortalized lines (MG63 and U2OS), Gai3 depletion, by shRNA and CRISPR/Cas9 strategies, robustly suppressed cell viability, proliferation and migration, while provoking GI-S arrest and apoptosis activation. Conversely, Gai3 overexpressing ectopically can accelerate proliferation and migration of OS cells. In OS cells, Gai3 immunoprecipitated with VEGFR2, FGFR, PGDFR and EGFR, mediating downstream cascade transduction. Akt-mTOR activation in primary OS cells was potently inhibited by Gai3 shRNA, knockout or dominant negative mutation, but augmented after Gai3 overexpression. In vivo studies showed that Gai3 shRNA AAV (adeno-associated viruses) intratumoral injection largely inhibited the growth of subcutaneous xenografts of primary OS cells. Moreover, the growth of the Gai3-knockout primary OS xenografts was much slower than that of OS xenografts with empty vector. In Gai3-depleted OS xenografts tissues, Gai3 downregulation and Akt-mTOR inactivation were detected. Taken together, overexpressed Gai3 mediates RTK-Akt signaling to drive OS progression.
\end{abstract}

Key words: Osteosarcoma; Gai3; multiple receptor tyrosine kinase; Akt-mTOR; Signaling

\section{Introduction}

For the advanced osteosarcoma (OS) patients with metastatic, recurrent or therapy-resistant OS, the prognosis is poor [1]. Further exploring the novel targeted therapeutics for OS is thus extremely important [2-7]. Due to various gene mutations, overexpression and/or over-activation of multiple receptor tyrosine kinases (RTKs) and its downstream oncogenic cascades are essential for initiation, progression and therapy-resistance of OS [4, 8, 9]. Several RTKs, including VEGFR, PDGFR, RET, EGFR and IGFR, as well as KIT and FGFR, are key drivers for the cancerous behaviors of OS [4, 8, 9]. Concurrent 
activation of multiple RTKs shall provoke sustained activation of oncogenic cascades, causing persistent cancer growth $[8,9]$. Therefore, targeting one or few RTKs, using genetic methods and/or pharmacological inhibitors, could only achieve minimal anti-OS efficiency $[8,9]$. The novel stragies targeting multiple RTKs simultaneously should achieve better anti-OS outcome $[8,9]$.

The inhibitory guanine nucleotide regulatory proteins, Gai proteins, consists of three subunits, including Gai1, Gai2 and Gai3 [10]. It is known that GPCRs ( $G$ protein-coupled receptors) binding to Gai proteins and the $\beta$ and $\gamma$ complexes will hinder adenylate cyclase, causing cyclic AMP (cAMP) depletion [10]. Such actions would be reversed by pertussis toxin [11]. Few studies have explored the expression, function and potential signaling mechanism of Gai proteins in OS. Pine et al., have shown that Gai proteins are important for the agonist-induced cAMP production in osteosarcoma cells that were derived from rat [12]. Wang et al., showed that pertussis toxin can inhibit bradykinininduced $\mathrm{Ca}^{2+}$ mobilization in MG63 OS cells [13].

Our group has identified an essential role of Gai proteins in transducing signals for multiple RTKs [14-20]. We found that EGFR-induced Akt-mTOR activation was abolished after Gai1/3 double knockout (DKO) or silencing [20]. With VEGF stimulation, Gai1/3 associated with VEFGR2, promoting VEGFR2 endocytosis and downstream signaling activation [16]. Similarly, Gai1/3 mediated BDNF (brain-derived neurotrophic factor)-stimulated Akt-mTOR activation [17]. Therefore, by mediating signaling transduction of multiple RTKs, Gai proteins could be important oncogenic genes and therapeutic targets for human cancer [14, 18, 21]. Indeed, we have previously shown that Gai proteins are upregulated in glioma and gastric cancers, required for cancer growth [14, 18, 21]. The current study explored Gai3 expression and potential functions in human OS.

\section{Methods}

\section{Ethics}

The protocols of the present study were reviewed and approved by the Ethics Committee of Soochow University and were in according to the principle of Helsinki declaration.

\section{Reagents}

LY294002 was from Sigma Aldrich (St Louis, M.O.). The antibodies were described in our previous studies [18]. All the primers, sequences, constructs and virus were synthesized by Shanghai Genechem Co. (Shanghai, China), or mentioned otherwise.

\section{Cells and tissues}

The immortalized OS lines, MG63 and U2OS, as well as the established hFOB1.19 osteoblastic cells were cultivated as described [22-24]. Patient-derived primary OS patients, namely pOS-1 and pOS-2, were described previously [22, 23]. Patient-derived human osteoblasts (pOsteoblasts) were differentiated and cultured using the previously-descried protocols [25, 26]. The human tissues, including the OS tumor tissue specimens and the adjacent normal bone tissue specimens [22, 23], were obtained from the writteninformed consent OS patients who were all administrated at the Affiliated Hospitals of Soochow University.

\section{Gene detection}

Protein detection by Western blotting, RNA assays by qRT-PCR and co-immunoprecipitation (co-IP) examining protein-protein interaction were extensively described early [17, 20]. When necessary, lysates from the same set of the experiment were detected in the parallel gels to test different proteins. The primers were described early [16].

\section{Gai3 shRNA}

The lentivirus-encoded Gai3 shRNAs and in vitro cell infection were described early [16]. Stable cells were selected by puromycin-containing complete medium (with FBS) for additional 96h. Gai3 silencing (with over 95-98\% knockdown efficiency) in the stable cells was always verified. The control cells were infected with non-sense scramble control shRNA lentivirus ("sh-C") [16]. For the in vivo studies, the Gai3 shRNA sequence or shC sequence was sub-cloned into an established adenoviral vector, adeno-associated virus 9/AAV9 construct (Genechem). Through Lipofectamine 3000 the construct was thereafter transfected to HEK-293T cells, and the shRNA-expressing AAV virus was generated and was injected to xenograft tumors.

\section{CRISPR/Cas9-induced Gai3 knockout (KO)}

OS cells were transfected with Cas9-expressing construct (Genechem) by Lipofectamine 3000 (Invitrogen, Shanghai, China) to establish stable cells. Next, the lenti-CRISPR/Cas-9 Gai3 KO construct [14, 16], was transduced to Cas9-expressing OS cells, with stable cells established by using puromycincontaining medium for additional 96h. Goi3 KO screening was carried out and thereafter the Gai3 KO cells were eventually established. The control OS cells were with a lenti-CRISPR/Cas-9 empty vector with non-sense small guide RNA (“Cas9-C”).

Gai3 overexpression and dominant negative mutation, stable cells establishment and verification 
were described in our previous studies $[14,16]$.

\section{Constitutively-active mutant Akt I}

OS cells were infected with the constitutivelyactive Akt1 (caAkt1, S473D)-expressing adenovirus (provided by Dr. Li $[27,28]$ ) for $48 \mathrm{~h}$. Puromycin was thereafter added for $96 \mathrm{~h}$ to establish the stable OS cells, where expression of the caAkt1 was confirmed by Western blotting.

\section{Akt1/2 shRNA}

The commercial available Akt1/2 shRNA lentiviral particles (sc-43609-V, Santa Cruz Biotech) were added and transfected to cultured OS cells. After $48 \mathrm{~h}$, cells were cultured and selected in puromycincontaining medium for another $96 \mathrm{~h}$. Akt $1 / 2$ silencing was always examined.

\section{Cellular functional studies}

The cell viability detection by cell counting kit-8 (CCK-8), the EdU nuclear staining assaying of cell proliferation, propidium iodide (PI)-FACS, "Transwell" assays were carried out using the previously described protocols [18, 21, 25, 29-33].

\section{Apoptosis detection}

Apoptosis-related assays, including the TUNEL nuclear staining, 7-AAD and Annexin V double staining, caspase-3/-9 activities measurement, and ELISA testing the cellular ssDNA (single strand DNA) contents were described early [21, 25, 31-33].

\section{Xenograft studies}

The primary pOS1 cells (five million cells in every mouse) were subcutaneously (s.c.) injected to the nude mice (18.5-19.5g, half female and half male, please refer to our previous studies [22, 23]). Tumor-bearing mice were then subject to the designated treatments. Tumor volumes [(length $\times$ width $\left.^{2}\right) / 2$ ] and animal body weights were weekly recorded. Soochow University's Ethics Committee and Institutional Animal Care and Use Committee (IACUC) reviewed and approved the protocols of animal studies.

\section{Statistical analyses}

Statistical analyses were described previously $[22,23]$. The numerical data in the bar graphs indicated the mean and standard deviation (S.D.). $P$-values $<0.05$ were statistically significant.

\section{Results}

\section{In human OS Gai3 is upregulated}

The Cancer Genome Atlas (TCGA) database (available on the public domain https://portal.gdc. cancer.gov) was first consulted to analyze Goi3 transcripts in human sarcoma tissues. Total 264 samples (HTSeq-FPKM) were collected, including the two normal specimens and the 262 sarcoma specimens. As shown, Goi3 transcripts in the retrieved sarcoma tissue specimens were higher than those in the retrieved normal tissue specimens (Figure 1A). The Kaplan-Meier survival, Figure 1B, verified that high Goi3 expression in sarcoma patients was correlated with a poor prognosis $(\mathrm{HR}=1.73, \boldsymbol{P}=$ 0.008 ). Subgroup analysis by different clinical features demonstrated that Goi3-high expression was associated with a poor prognosis in male sarcoma patients $(\boldsymbol{P}=0.003$, Figure $1 C)$, age $\leq 60(\boldsymbol{P}=0.048$, Figure 1D), Tumor depth: deep $(P=0.009$, Figure 1E).

Next, we tested Gai3 expression in local OS tissues. As previously described [22, 23], OS tissue specimens and matched adjacent bone normal tissue specimens were retrieved from 10 different OS patients. Figure 1F showed that Gai3 mRNA in the OS tissue specimens (" $\mathrm{T}$ ") was more than six-fold higher than that in the adjacent normal bone tissues ("N"). Gai3 protein expression was tested as well. In the OS tissues of four representative OS patients (Patient $\# 1 / \# 2 / \# 3 / \# 4$ ), protein expression of Gai3 was significantly elevated (Figure 1G). When combining all blotting results of the ten sets tissues, Gai3 protein was found to be significantly upregulated in the OS tissues $(P<0.001$ versus "N" tissues, Figure $\mathbf{1 H})$. The immunohistochemistry (IHC) staining assay results, in Figure 1I, verified Gai3 protein elevation in OS tumor tissues (Figure 1I), While low expression of Gai3 was detected in the adjacent normal tissue specimens (Figure 1I).

The expression of Gai3 in different human OS cells was examined. Patient-derived primary OS cells (pOS-1/2, from two different OS patients), as well as the immortalized cells (MG63 and U2OS lines), were cultured. The expression of Goi3 mRNA in different primary and immortalized OS cells was dramatically higher than that in hFOB1.19 human osteoblastic cells and patient-derived human osteoblasts ("pOsteoblasts") (Figure 1J). In addition, Gai3 protein upregulation was also shown in different primary and established OS cells (Figure 1K). Taken together, in OS tissues and cells Gai3 is upregulated.

\section{Gai3 silencing exerts anti-tumorigenic activity in cultured OS cells}

pOS-1 primary cells were separately transfected with two different Gai3 lentiviral shRNAs, sh-Gai3seq1/sh-Gai3-seq2 [22, 23]. After puromycin-induced selection, Gai3-silenced stable OS cells were established. Gai3 mRNA expression decreased over 90-95\% in sh-Gai3-expressing stable pOS-1 cells 
(Figure 2A). Expression of Gai1 and Gai2 mRNA was unaffected (Figure 2A). Gai3 protein silencing was detected in the stable pOS-1 cells with Gai3 shRNAs. Gai1 and Gai2 protein expression was again unchanged (Figure 2B).

CCK-8 assays showed that Gai3 shRNA potently decreased pOS-1 cell viability (CCK-8 OD, Figure 2C). Moreover, Gai3 shRNA potently inhibited pOS-1 cell proliferation, as the ratio of EdU positively stained nuclei was robustly decreased in sh-Gai3-expressing pOS-1 cells (Figure 2D). The PI-FACS assays were employed to test cell cycle progression. The ratio of G1-phase cells was significantly increased in pOS-1 cells expressing Gai3 shRNA (Figure 2E), where S-phase pOS-1 cell percentage was decreased (Figure 2F). These results implied that the designated Gai3 shRNAs induced G1-S arrest in primary OS cells. The "Transwell" results demonstrated that Gai3 shRNA dramatically inhibited pOS-1 cell in vitro migration (Figure 2G). The scramble control shRNA, or shC, did not significantly alter Gail/2/3 expression (Figure 2A-B) and affect OS cellular behaviors (Figure 2C-G).
A.

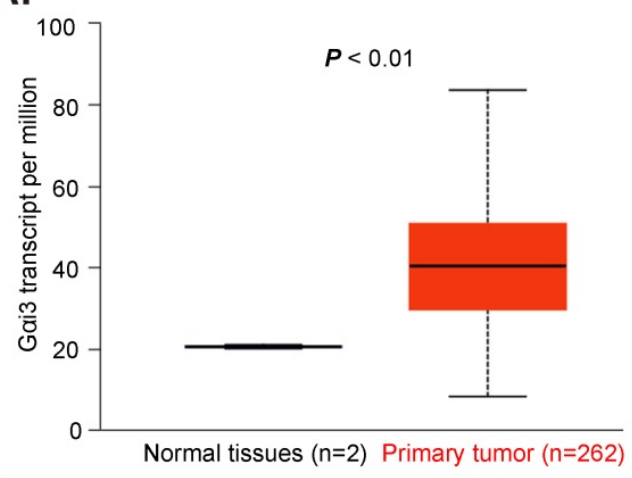

C.

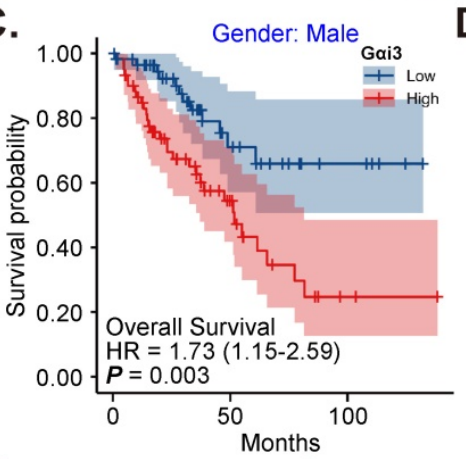

F.

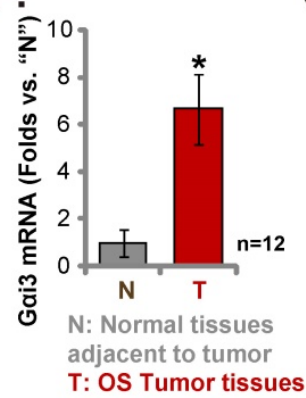

G.

D.

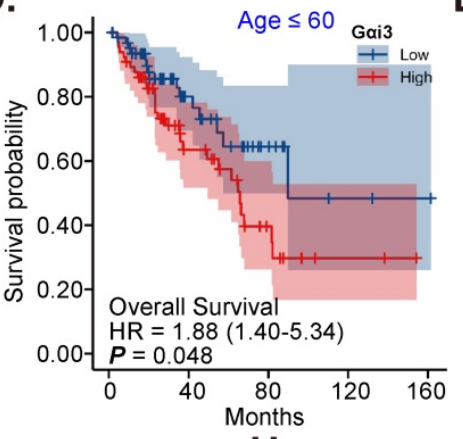

$\mathrm{H}$.

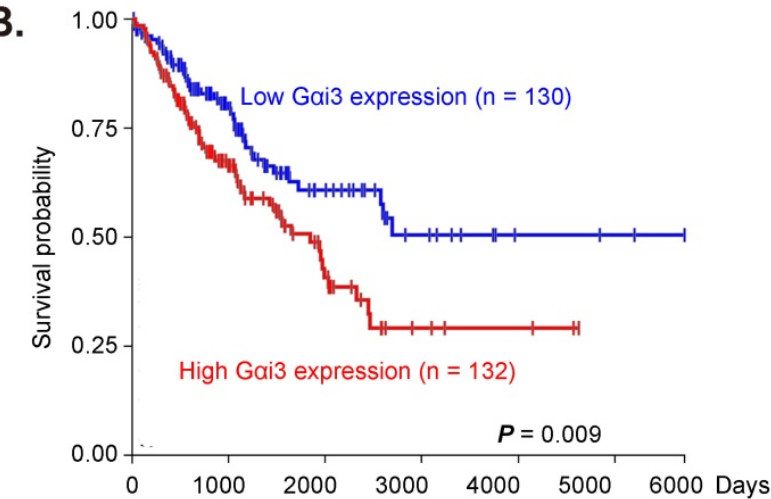

E.

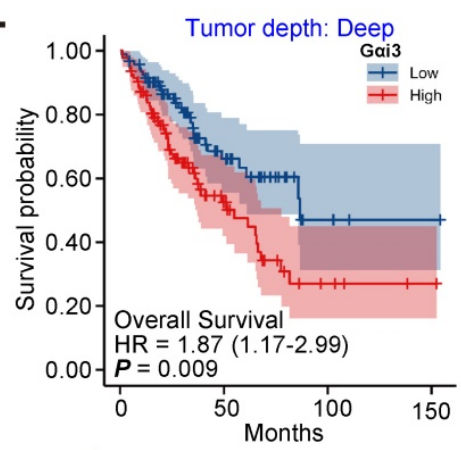

I.

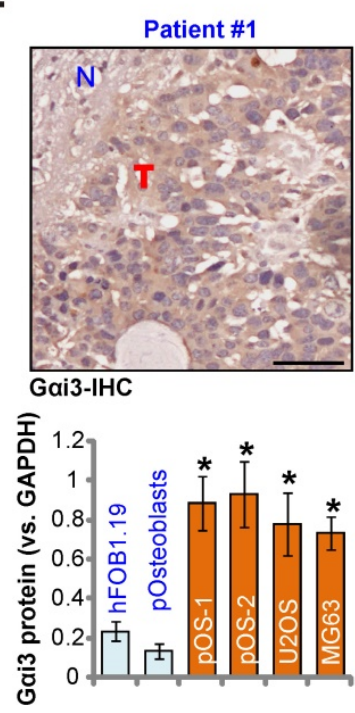

Figure 1. In human OS Gai3 is upregulated. TCGA cohorts show Goi3 mRNA transcripts in 262 sarcoma cases ("Primary Tumor") and two normal tissue cases (A). The Kaplan Meier Survival curve of Gai3-low $(n=130$, blue) and Gai3-high $(n=132$, red) sarcoma patients was presented (B). Subgroup analyses, based on the different clinical features of the OS patients, were performed as well (C-E). The expression of Gai3 (both mRNA and protein) in twelve (n = 12) pairs of OS tumor tissue specimens ("T") and adjacent normal bone tissue specimens ("N") was tested (F-H). The representative human tissue Gai3 IHC were presented as well (I). The expression of Gai3 mRNA and protein in the mentioned cells was measured $(\mathbf{J}-\mathbf{K}) . * \mathbf{P}<0.05$ versus " $\mathrm{N}$ " tissues $(\mathbf{F}$ and $\mathbf{H})$ or hFOB1.19 cells $(\mathbf{J}$ and $\mathbf{K})$. Scale bar $=100 \mu \mathrm{m}(\mathbf{I})$. 
A.

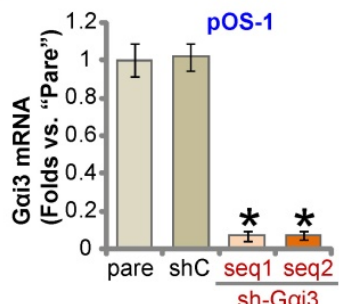

D.

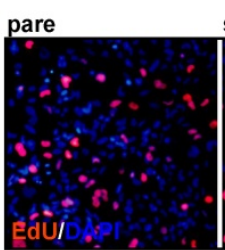
shc

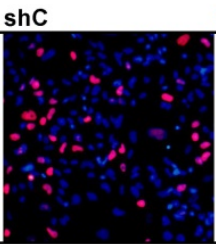

Cell proliferation

G. pare

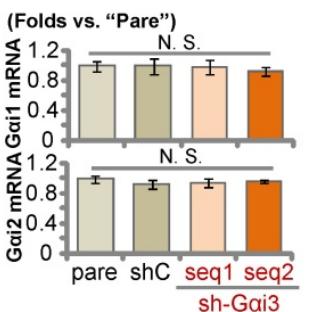

B.

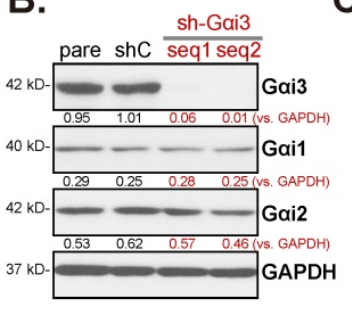

C.

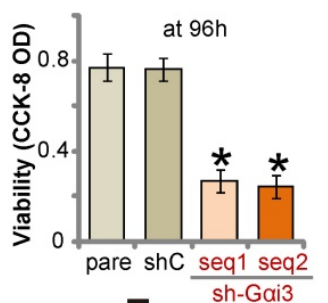

E.
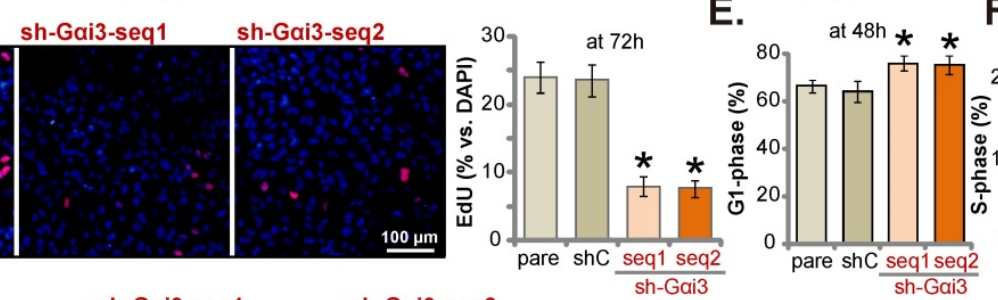

$\mathrm{F}$.
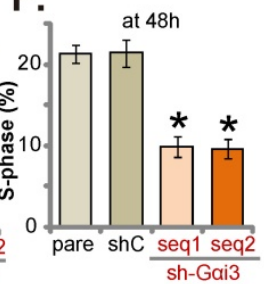
sh-Gai3-seq2

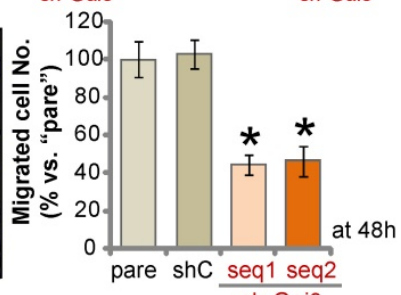

$\mathrm{H}$.

$$
\text { Cell migration }
$$

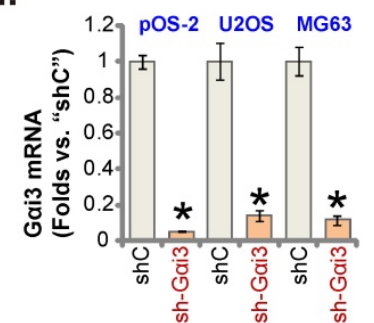

L.

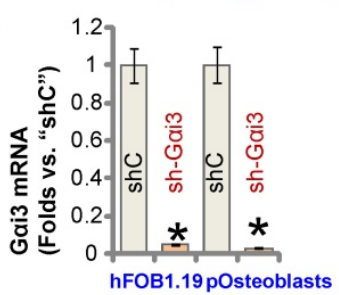

I.

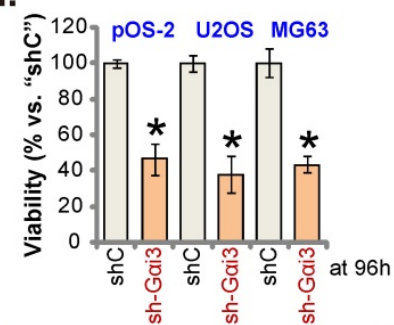

M.

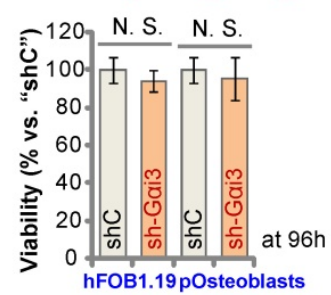

J.

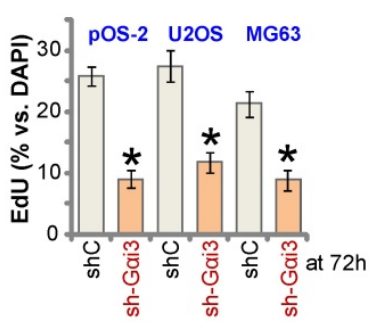

N.

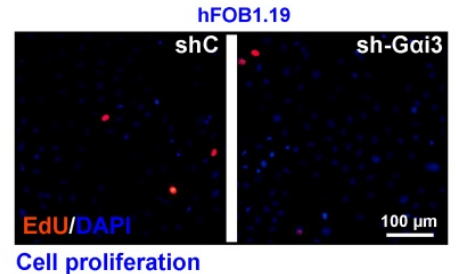

K.

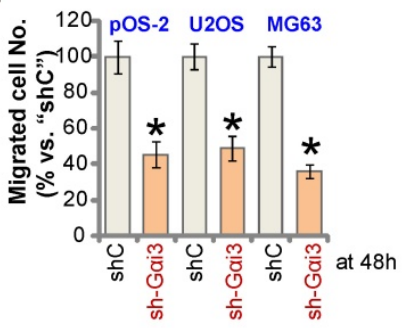

Figure 2. Gai3 silencing exerts anti-tumorigenic activity in cultured OS cells. Patient-derived primary human OS cells ("pOS-1/2"), the immortalized OS lines (MG63 and U2OS), the immortalized hFOB1.19 cells or patient-derived primary osteoblasts ("pOsteoblasts") were stably transduced with the designated Gai3 lentivirus shRNA (sh-Gai3-seq1/sh-Gai3-seq2, with two different sequences) or the non-sense control shRNA ("shC"), the expression of indicated mRNAs and proteins were examined (A, B, $\mathbf{H}$ and $\mathbf{L}$ ). After culturing for the designated hours, CCK-8 viability (C, I and $\mathbf{M}$ ), and cell proliferation (testing the ratio of EdU positively stained nuclei , D, J and $\mathbf{N}$ ), as well as cell cycle progression (PI-FACS assays, results quantified in $\mathbf{E}$ and $\mathbf{F}$ ) and cell migration ("Transwell" assays, $\mathbf{G}$ and $\mathbf{K}$ ) were tested by the listed assays. "pare" indicated the parental control OS cells. $* \boldsymbol{P}<0.05$ versus "shC" group. "N. S." indicated no statistical difference $(\boldsymbol{P}>0.05, \mathbf{A}, \mathbf{M}$ and $\mathbf{N})$. Each single experiment was repeated for five times. Scale bar $=100 \mu \mathrm{m}(\mathbf{D}, \mathbf{G}$ and $\mathbf{N})$.

Next, OS cells that were derived from another OS patient, pOS2 [22, 23], and the immortalized lines (MG63 and U2OS), were stably transduced with the lentiviral Gai3 shRNA (sh-Gai3-seq1, "sh-Gai3"). As shown, the designated shRNA resulted in robust Gai3 mRNA downregulation in both primary and immortalized OS cells (Figure 2H). Gai3 shRNA largely suppressed CCK-8 viability (Figure 2I), proliferation (the ratio of EdU positively stained nuclei reduction, Figure 2J) and migration (Figure 2K) in pOS2 primary cells and established lines.
The potential effect of Gai3 shRNA on the Gai3-low human osteoblasts (see Figure 1J-K) was tested as well. hFOB1.19 osteoblastic cell line or patient-derived primary osteoblasts (pOsteoblasts) were stably transduced with the lentiviral sh-Gai3seq1, causing dramatic Gai3 mRNA downregulation ("sh-Gai3", Figure 2L). Interestingly, in hFOB1.19 cells and pOsteoblasts, Gai3 shRNA failed to significantly inhibit CCK-8 viability (Figure $\mathbf{2 M}$ ) and cell proliferation (by measuring EdU positively stained nuclei ratio, Figure $\mathbf{2 N}$ ), supporting a cancer cell-specific effect by Gai3 shRNA. 

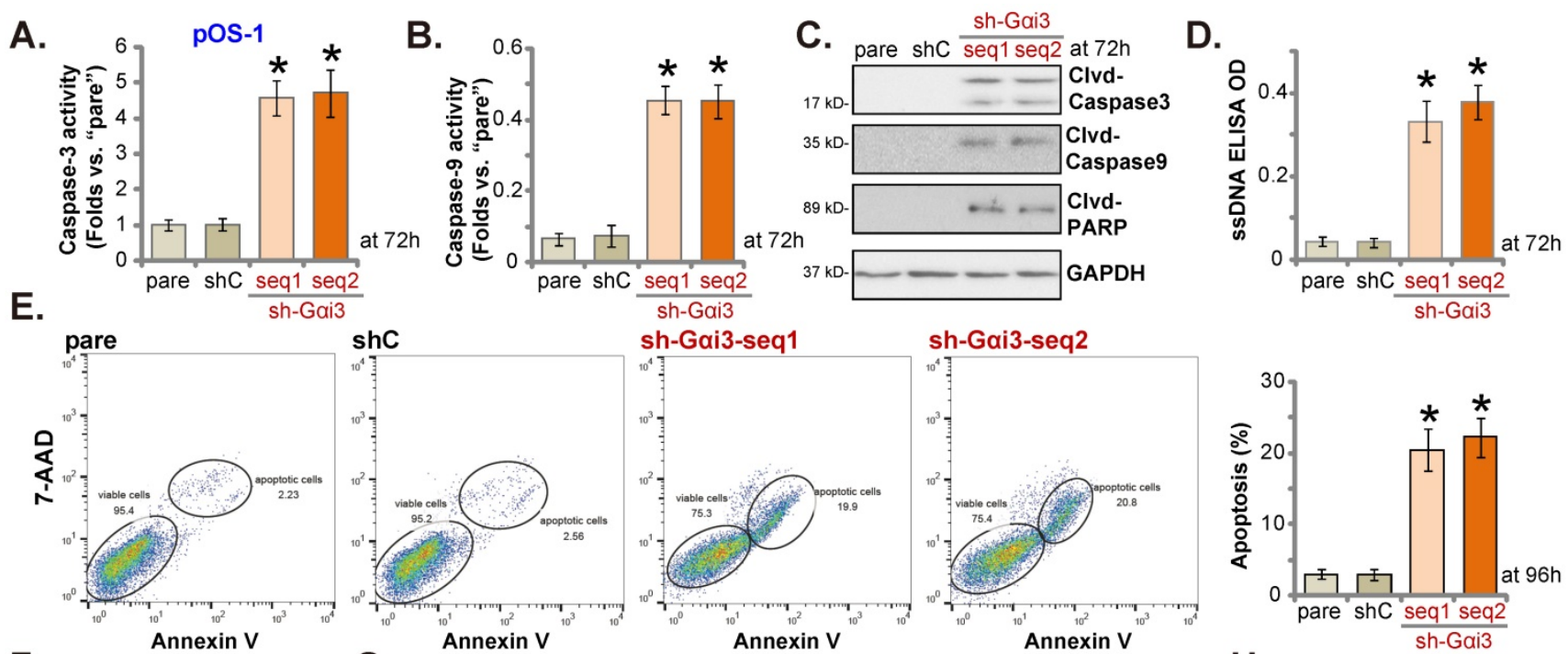

F.
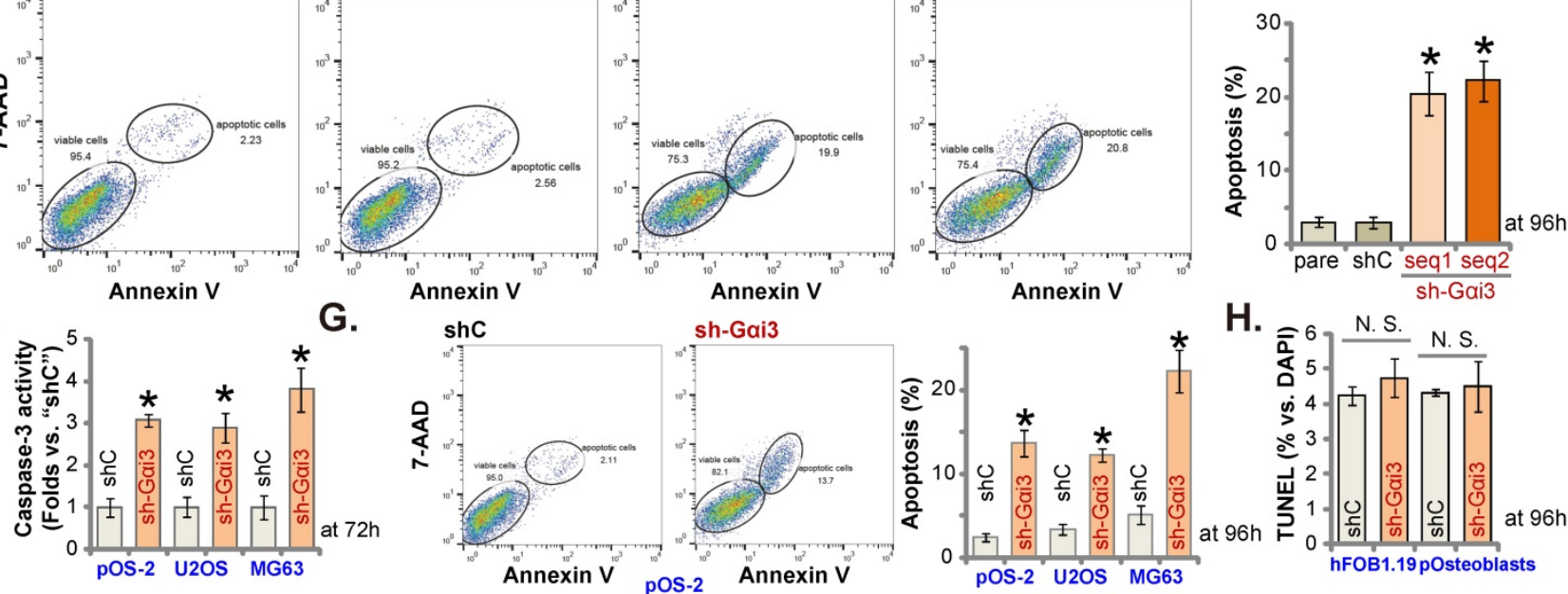

Figure 3. Gai3 silencing provokes apoptosis activation in OS cells. Patient-derived primary human OS cells ("pOS-1/2"), the immortalized OS lines (MG63 and U2OS), the immortalized hFOB1.19 cells or patient-derived primary osteoblasts ("pOsteoblasts") were stably transduced with the designated Gai3 lentivirus shRNA (sh-Gai3-seq1/sh-Gai3-seq2, two different sequences) or the non-sense control shRNA ("shC"). After culturing for the designated hours, the caspase-PARP activation was tested (A-C, F), with DNA breaks tested by ssDNA ELISA assays (D); Cell apoptosis was examined by Annexin V-7-AAD double staining FACS (E and G) and nuclear TUNEL staining (results quantified in $\mathbf{H}$ ) assays. All blotting data in this Figure were repeated five times. "pare" indicated the parental control OS cells. *P< 0.05 versus "shC" group. "N. S." indicated no statistical difference $(\boldsymbol{P}>0.05, \mathbf{H})$. Each single experiment was repeated for five times.

\section{Gai3 silencing provokes apoptosis activation in OS cells}

Since Gai3 silencing induced viability reduction, growth arrest, proliferation inhibition, we therefore tested its potential function on apoptosis in OS cells. Results showed that the relative activities of caspase- 3 and caspase-9 (Figure 3A and $\mathbf{B}$ ) were augmented in sh-Gai3-expressing stable pOS-1 cells. Figure 3C showed that Gai3 shRNA induced cleavages of caspase-3, PARP and caspase-9 in pOS-1 primary cells. Confirming increased DNA breaks in pOS-1 cells with Gai3 shRNA, we showed that ssDNA contents were dramatically increased (tested by the ELISA assays, Figure 3D). Moreover, FACS assay results in Figure 3E showed that Gai3 silencing increased the pOS-1 cell number with Annexin V-7-AAD double positive staining, confirming apoptosis activation. As expected, shC did not provoke caspase-apoptosis activation in the primary pOS-1 cells (Figure 3A-E).

In pOS-2 cells and immortalized lines (MG63 and U2OS), Gai3 shRNA (sh-Gai3-seq1, "sh-Gai3", see Figure 2) similarly augmented the relative caspase-3 activity (Figure 3F). Apoptosis induction was observed as well in the sh-Gai3-seq1-expressing
pOS-2 cells and immortalized cell lines, evidenced by the significantly increased Annexin V-7-AAD double staining (Figure 3G). Conversely, in the osteoblastic cell line hFOB1.19 and pOsteoblasts, shRNA-induced silencing Gai3 ("sh-Gai3", see Figure 2) failed to significantly induce apoptosis (by quantifying TUNEL-positively stained nuclei, Figure $3 \mathbf{H}$ ).

\section{Gai3 knockout potently inhibits OS cell progression in vitro}

Next, a previously-described CRISPR/Cas9Gai3-KO-puro construct $[15,16]$ was transduced to the Cas9-expressing pOS-1 primary cells. Thereafter, single stable pOS-1 cells with the Gai3 KO construct, or the ko-Gai3 cells, were established after Goi3 KO screening, and Goi3 mRNA and protein (Figure 4A) depletion was detected. Gai3 KO largely decreased CCK-8 viability (Figure 4B) and inhibited cell proliferation (the ratio of EdU positively stained nuclei decreasing, Figure 4B) and migration (Figure 4C) in pOS-1 cells. On the contrast, caspase- 3 and caspase-9 activities (Figure 4D) were augmented in ko-Gai3 pOS-1 cells, where caspase-3, PARP and caspase-9 cleavages were induced (Figure 4D). In addition, apoptosis induction was detected in the ko-Gai3 pOS-1 cells, as the ratio of TUNEL-positively 
stained nuclei was increased significantly (see the quantified results in Figure 4E).

The Gai3-KO construct was employed to knockout Gai3 in primary pOS-2 cells and immortalized lines (MG63 and U2OS), and stable cells established ("ko-Gai3") after screening (Figure 4F). As shown, Gai3 KO inhibited cell proliferation (the ratio of EdU positively stained nuclei reduction, Figure 4G) and in vitro migration (Figure $\mathbf{4 H}$ ) in pOS-2 and established OS cells. Increased TUNEL-positive nuclei ratio confirmed apoptosis activation in ko-Gai3 pOS-2 cells and immortalized lines (Figure 4I). Together, Gai3 KO, by the CRISPR/Cas9 strategy, resulted in profound anti-OS cell activity.

\section{Further promoting OS cell growth by Gai3 ectopic overexpression}

Next, a Gai3-expressing lentiviral construct (see our previous studies [14, 16]) was transduced to pOS-1 cells. Following selection Gai3-overexpressed stable pOS-1 cells were thereafter established: namely "OE-Gai3-sL1" and "OE-Gai3-sL2" (two selections). Gai3 mRNA and protein expression levels (Figure 5A and B) were robustly elevated in the OE-Gai3 pOS-1 cells. Gai3 overexpression accelerated pOS-1 cell proliferation (the ratio of EdU positively stained nuclei increasing, Figure 5C) and in vitro migration ("Transwell" assays, results quantified in Figure 5D).
In pOS-2 cells and the immortalized lines (MG63 and U2OS), ectopic overexpression of Gai3 using the same construct ("OE-Gai3", Figure 5E) enhanced cell proliferation (the ratio of EdU positively stained nuclei increasing, Figure $5 \mathrm{~F}$ ) and in vitro migration (see the quantified results in Figure 5G). Therefore, these results again supported the key cancerpromoting function of Gai3 in OS cells.

\section{Gai3 immunoprecipitates with RTKs and is key to Akt-mTOR activation in OS cells}

Our previous studies have shown that Gai proteins associated with several oncogenic RTKs (EGFR, VEGFR2, TrkB, FGFR and KGFR), mediating downstream signaling activation [14, 16-21]. Co-IP (co-immunoprecipitation) assays, Figure 6A, demonstrated that Gai3 immunoprecipitated with VEGFR2, FGFR, PGDFR and EGFR in primary OS cells ("pOS-1/2") and immortalized U2OS cells. Moreover, the association between Gai3 and multiple RTKs (VEGFR2, FGFR, PGDFR and EGFR) was detected in the human OS tissues from three representative patients (Figure 6B). When testing downstream Akt-mTOR signaling, we showed that levels of phosphorylated-Akt (Ser-473) and phosphorylated-S6K (Thr-389) were dramatically decreased in pOS-1 cells bearing Gai3 shRNAs (Figure 6C). Moreover, CRISPR/Cas9-induced Gai3
A.

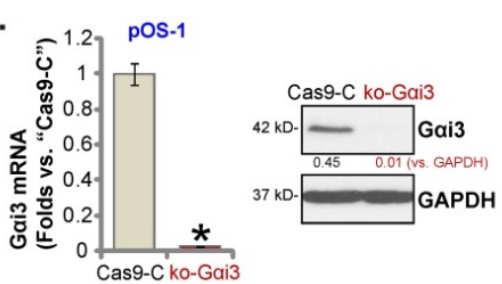

B.

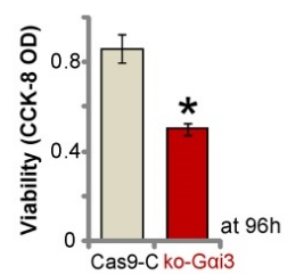

C. Cas9-C ko-Gai3

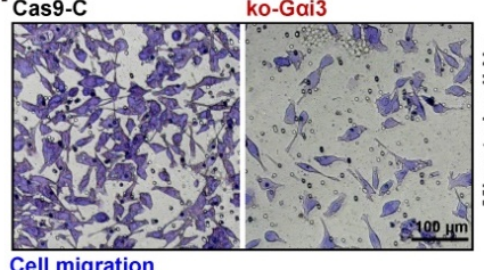

F.

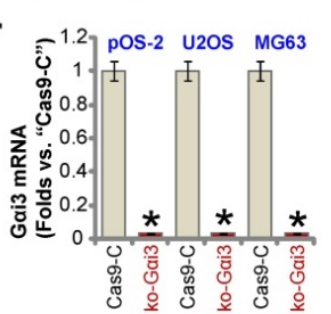

G.

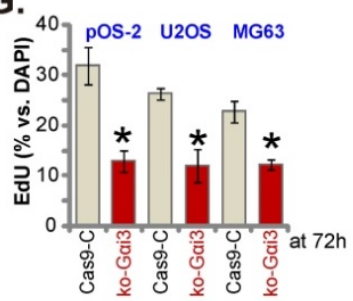

D.

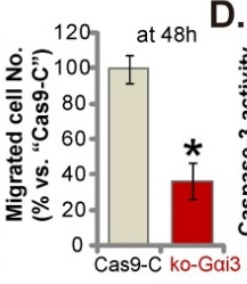

H.
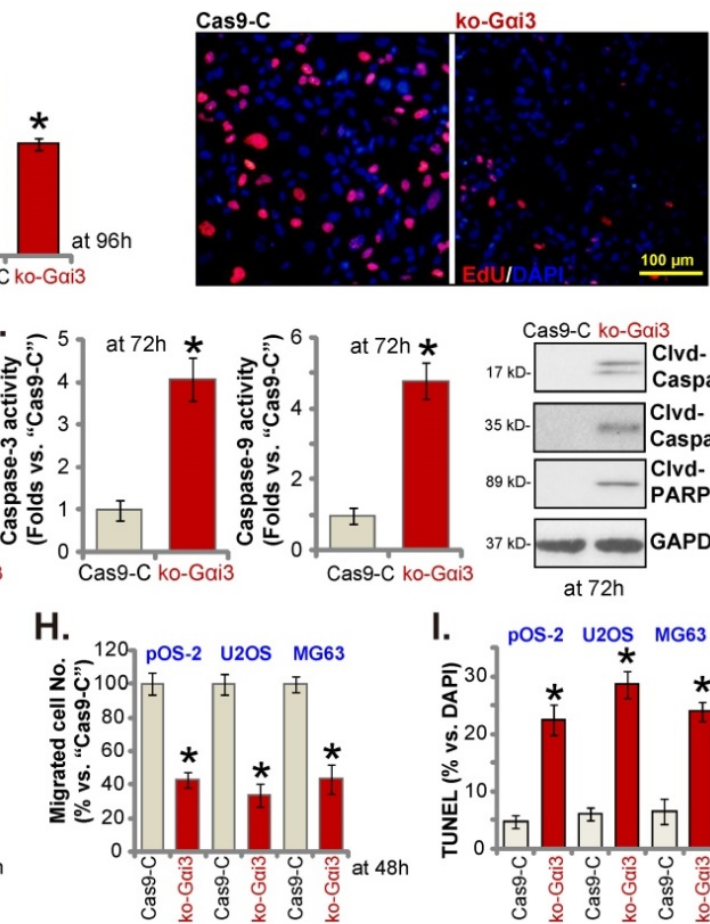

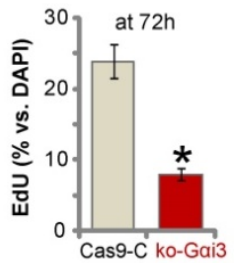

E.
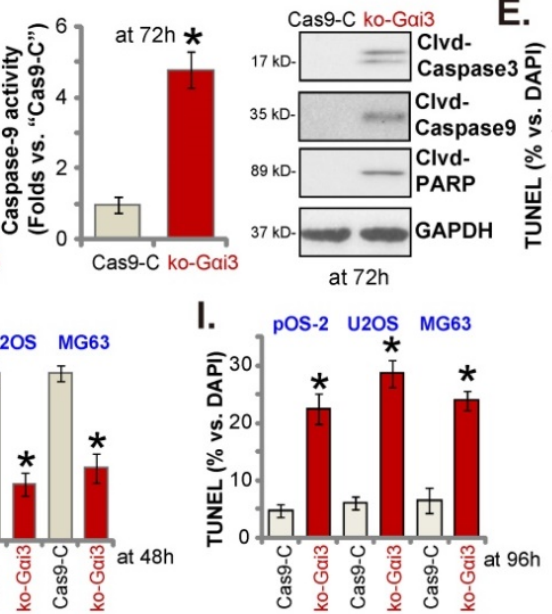

Figure 4. Gai3 knockout potently inhibits OS cell progression in vitro. Patient-derived OS cells ("pOS-1/-2") or immortalized OS lines (MG63 and U2OS), bearing the CRISPR/Cas9-Gai3-KO-puro construct ("ko-Gai3") or the corresponding vector ("Cas9-C"), were established, expression of listed mRNAs and proteins were examined (A and F); After culturing for the designated hours, CCK-8 viability (B), proliferation (testing the ratio of EdU positively stained nuclei , B and G), cell migration ("Transwell" assays, $\mathbf{C}$ and $\mathbf{H}$ ), caspase-PARP activation was tested (D), with cell apoptosis measured through quantifying the TUNEL-positively stained nuclei ratio (E and I). All blotting data in this Figure were repeated five times. *P $<0.05$ versus "Cas9-C" group. Each single experiment was repeated for five times. Scale bar $=100 \mu \mathrm{m}(\mathbf{B}$ and C). 
$\mathrm{KO}$ (see Figure 4) largely inhibited Akt-S6K phosphorylations in pOS-1 cells (Figure 6D). Notably, RTKs (FGFR, PGDFR and EGFR) expression and phosphorylation were unaffected by Gai3 shRNA
(sh-Gai3-seq1) or Gai3 KO (Figure 6D). Conversely, ectopic overexpression of Gai3 (see Figure 5) significantly increased Akt-S6K activation in pOS-1 cells (Figure 6E).
A.

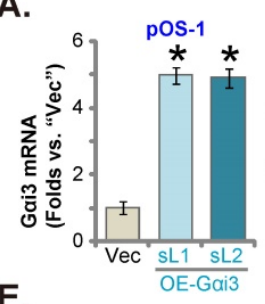

B.

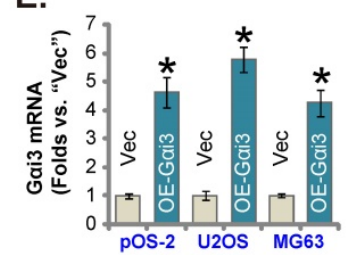

C.

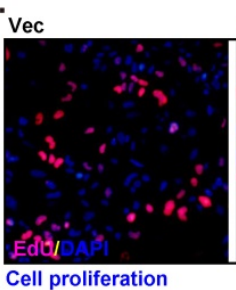

F. Cas9-C

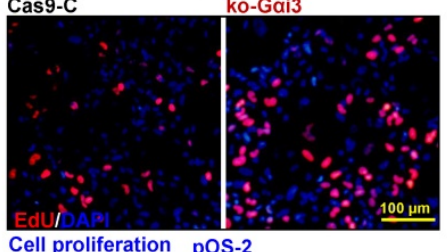

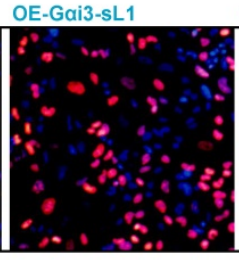

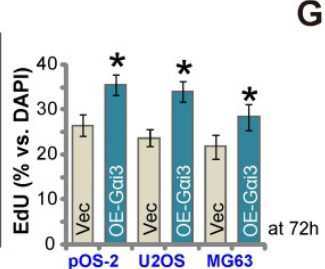

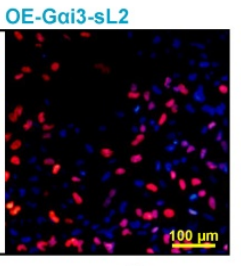

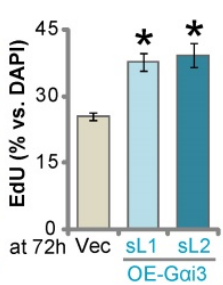

D.

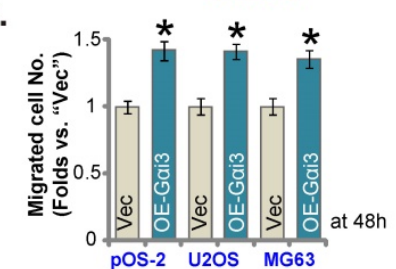

Figure 5. Further promoting OS cell growth by Gai3 ectopic overexpression. Patient-derived primary OS cells ("pOS-1/-2") or immortalized OS lines (MG63 and U2OS), bearing the lentiviral construct encoding wild-type Goi3 ("OE-Gai3") or the corresponding vector ("Vec"), were established and cultivated, expression of listed mRNAs and proteins were measured (A, B and E); After culturing for the designated hours, cell proliferation (testing the ratio of EdU positively stained nuclei , C and $\mathbf{F}$ ) and cell migration ("Transwell" assays, $\mathbf{D}$ and $\mathbf{G}$ ) were measured. All blotting data in this Figure were repeated five times. * $\boldsymbol{P}<0.05$ versus "Vec" group. Each single experiment was repeated for five times. Scale bar $=100 \mu \mathrm{m}(\mathbf{C}$ and $\mathbf{F})$.

A.
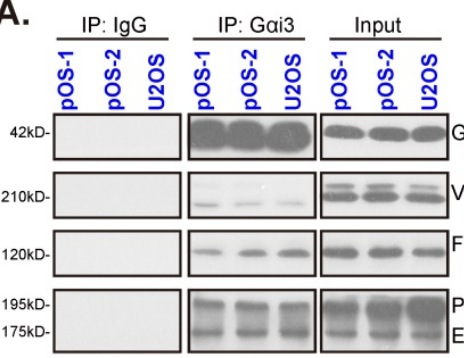

B.

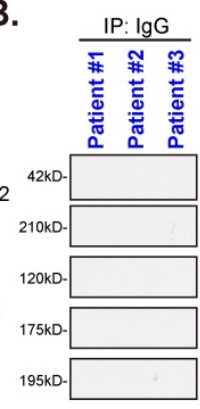

D.
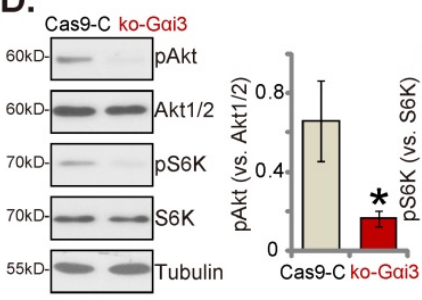

F.

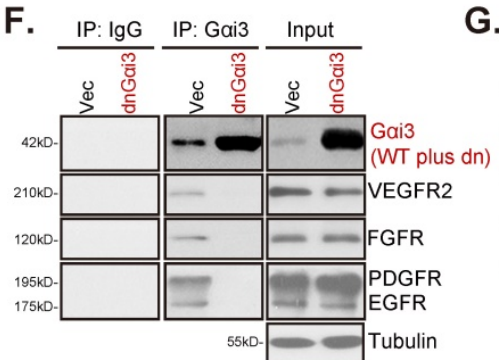

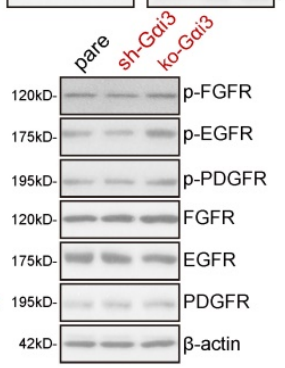

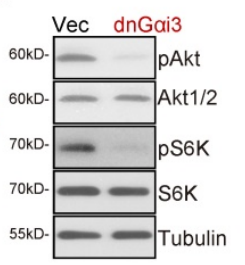

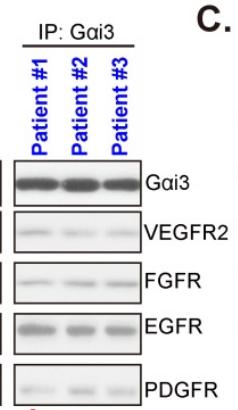

E.
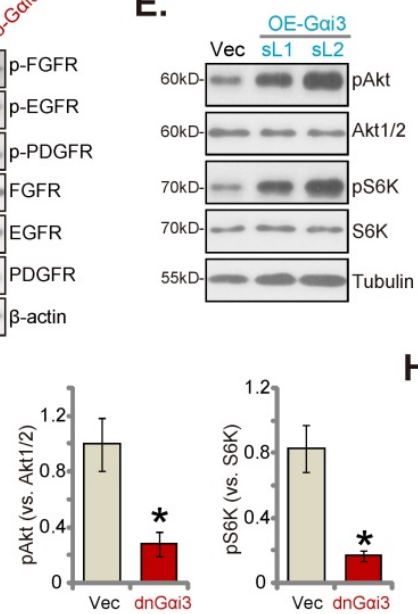

C. pos-1
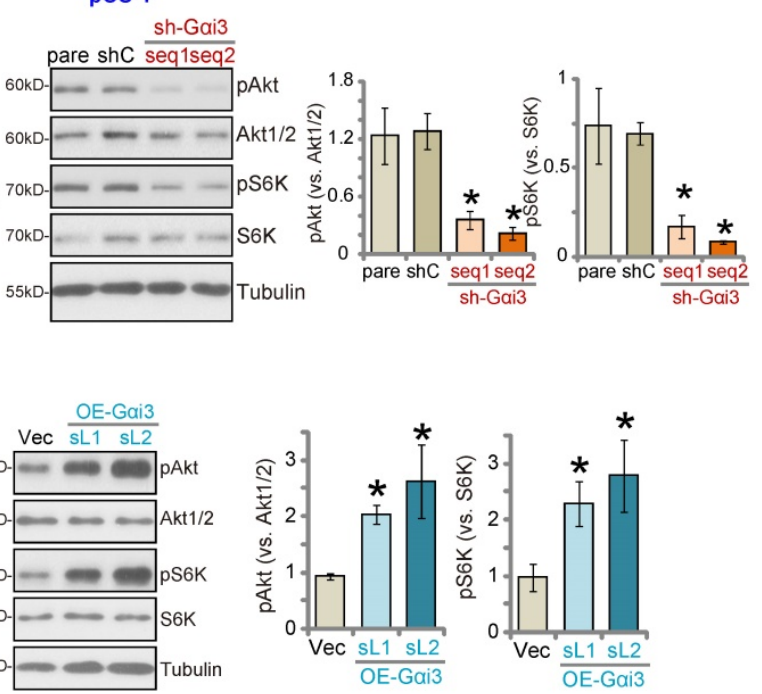

H.

I.
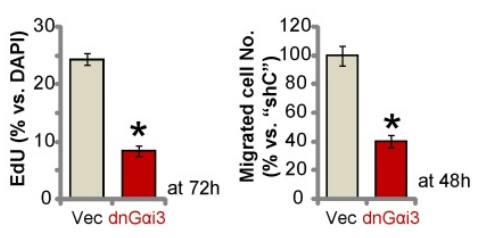

Figure 6. Gai3 immunoprecipitates with RTKs and is key to Akt-mTOR activation in OS cells. The association between Gai3 and the designated RTKs (VEGFR2, FGFR, PGDFR and EGFR) in patient-derived primary human OS cells ("POS-1/2") and U2OS line (A, cultured in FBS-containing medium for 5 min) as well as in OS tissues of the representative patients (B) was examined by the co-immunoprecipitation (Co-IP) assays. The pOS-1 primary cells stably expressing the Gai3 shRNA

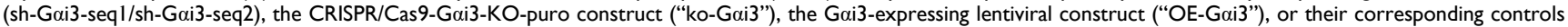
("shC", "Cas9-C" or "Vec") were established, and expression of listed proteins tested (C-E). The pOS-1 primary cells, stably expressing the lentiviral dominant negative Gai3 construct (dnGai3) or the empty vector ("Vec"), were established, the association between Gai3 and RTKs (VEGFR2, FGFR, PGDFR and EGFR) as well as their expression were examined (F and $\mathbf{G})$. After culturing for the designated hours, cell proliferation and migration were separately examined by EdU staining (H) and "Transwell" (I) assays. All blotting data in this Figure were repeated five times. "pare" indicated the parental control OS cells. *P< 0.05 versus "shC"/“Cas9-C"/“Vec" group. Each single experiment was repeated for five times. 
A.

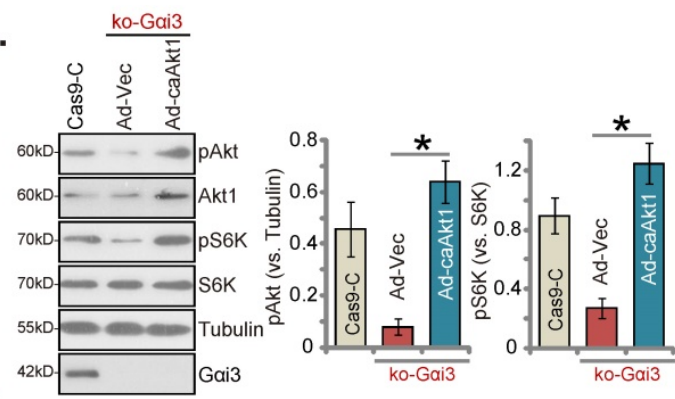

C.

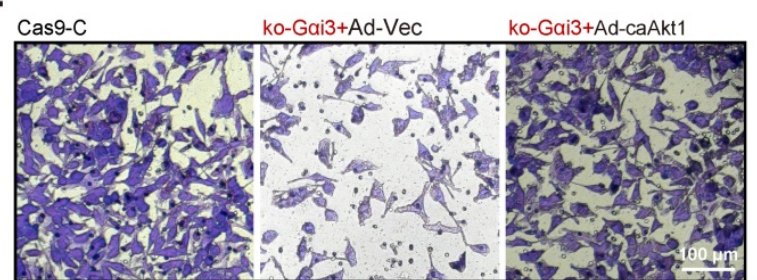

E.

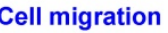

B.

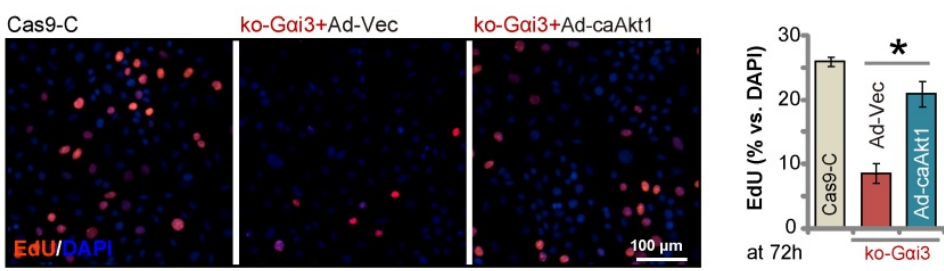

Cell proliferation

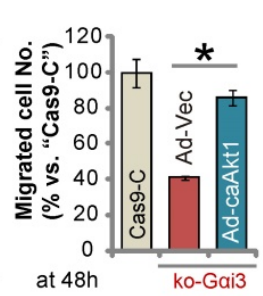

D.

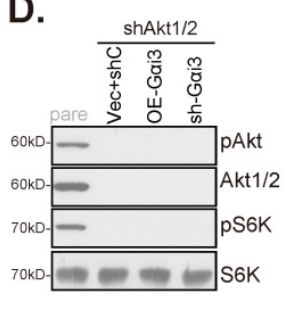

F.

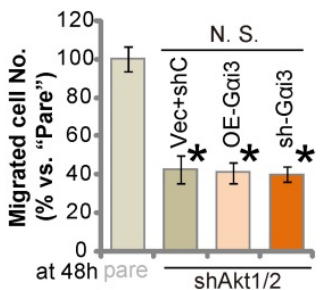

G.
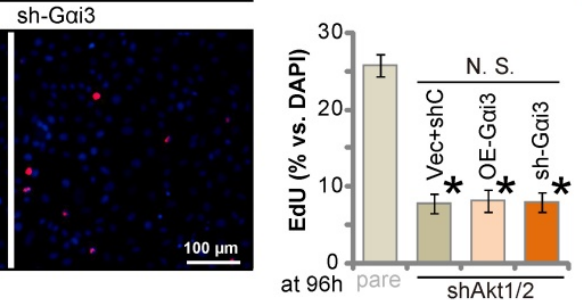

H.

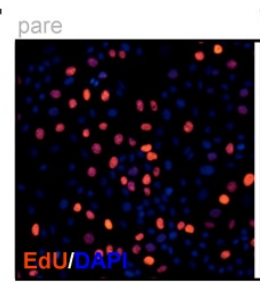

Cell proliferation

OE-Gai3-sL1 I.
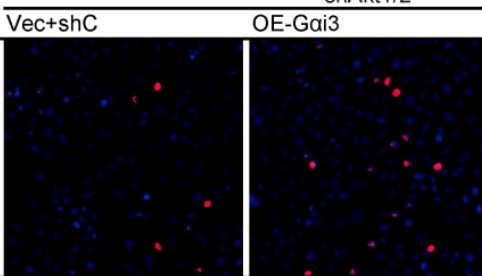

I.

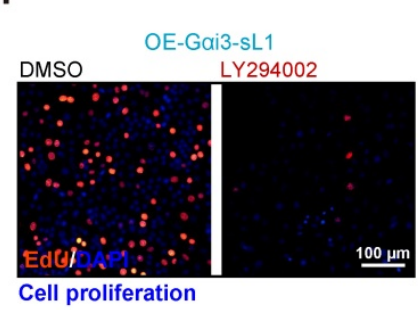

Cell proliferation

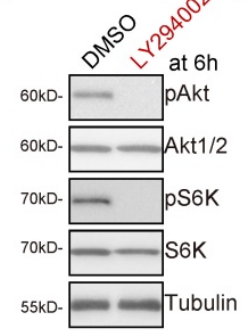

J.
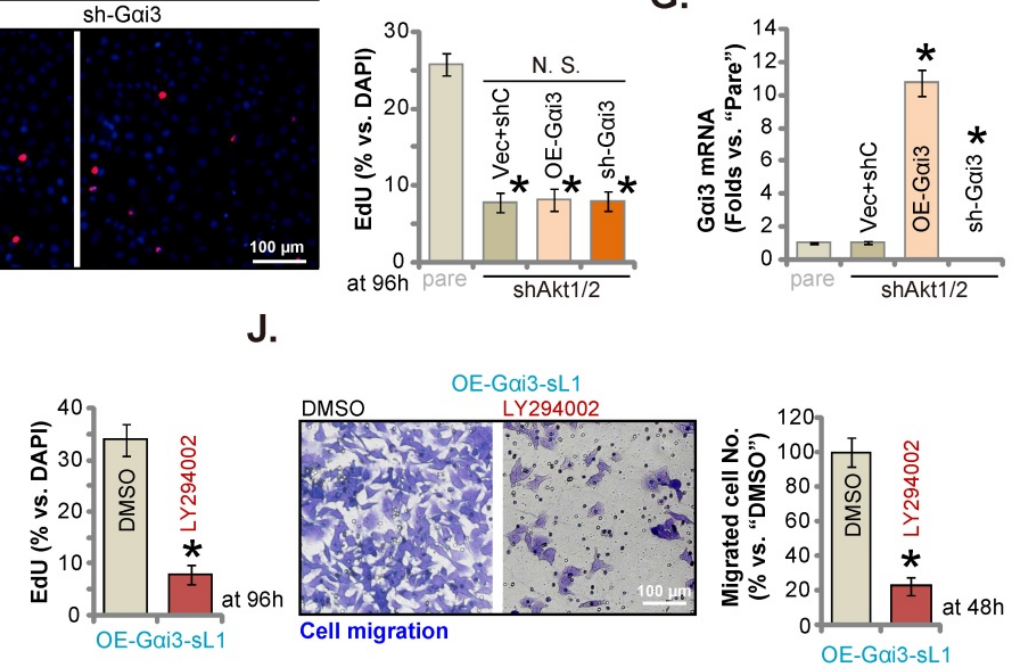

Figure 7. Akt-mTOR inhibition contributes to Gai3 depletion-induced anti-OS cell activity. The pOS-1 cells bearing the CRISPR/Cas9-Gai3-KO-puro construct ("ko-Gai3") were further infected with the constitutively-active Aktl adenovirus ("Ad-caAktl") or the adenovirus with the empty vector ("Ad-Vec"), control cells were with the CRISPR/Cas9 empty vector ("Cas9-C"), listed proteins were shown (A). Cells were cultured for designated hours, cell proliferation (B, EdU assays) and migration (C, "Transwell" assays) were tested. pOS-1 cells stably bearing the lentiviral Akt1/2 shRNA ("shAkt1/2") were further transduced with a wild-type Gai3 ("OE-Gai3") lentiviral construct, the lentiviral sh-Gai3-seql ("sh-Gai3") or their control construct ("Vec+shC"), stable cells were established. Gai3 mRNA and listed proteins were shown (D and G). After culturing for the designated hours, cell proliferation (E, by measuring EdU positively stained nuclei ratio) and migration (F) were measured. pOS-1 cells, bearing the lentiviral construct encoding wild-type Gai3 ("OE-Gai3-sL1") were treated with LY294002 (150 nM) or the vehicle control (0.1\% DMSO), and cultured for designated time periods, listed proteins were shown $(\mathbf{H})$, with cell proliferation $(\mathbf{I})$ and migration $(\mathbf{J})$ examined as well. All blotting data in this Figure were repeated five times. "pare" indicated the parental control OS cells. $* \boldsymbol{P}<0.05(\mathbf{A}-\mathbf{C})$. $* \boldsymbol{P}<0.05$ versus "pare" cells $(\mathbf{E}-\mathbf{G})$. $* \boldsymbol{P}<0.05$ versus "DMSO" $(\mathbf{I}$ and $\mathbf{J})$. "N. S." indicated no statistical difference $(\boldsymbol{P}>0.05, \mathbf{E}$ and F). Each single experiment was repeated for five times. Scale bar $=100 \mu \mathrm{m}$ (B, C, E, I and J).

To block Gai3-RTKs association, the lentiviral dominant negative (dn) Gai3 mutant construct was stably transduced into pOS-1 cells. The dnGai3 mutant will replace the conserved Gly $(G)$ residue with the Thr $(\mathrm{T})$ residue in the G3 box, thereby preventing Gai1/3 interaction with the associated proteins $[19,20]$. Results show that dnGai3 disrupted the association between Gai3 and multiple RTKs (VEGFR2, FGFR, PGDFR and EGFR) in pOS-1 cells (Figure 6F). Expression of RTKs was however unchanged (Figure 6F, "Input"). Importantly, dnGai3 largely inhibited Akt-S6K phosphorylations in pOS-1 primary cells (Figure 6G). The dnGai3 largely suppressed pOS-1 cell in vitro proliferation and migration, examined through the nuclear EdU staining (see the quantified results in Figure $\mathbf{6 H}$ ) and "Transwell" (Figure 6I) assays, respectively.

\section{Akt-mTOR inhibition contributes to Gai3 depletion-induced anti-OS cell activity}

To support that Akt-mTOR inhibition was the main mechanism of Gai3 depletion-caused anti-OS cell activity, we expressed the constitutively active Akt1 (caAkt1) [34] adenovirus ("Ad-caAkt1") that could rescue Akt and S6K phosphorylation in koGai3 pOS-1 cells (Figure 7A). Significantly, Ad-caAkt1 restored proliferation (by quantifying EdU-positively stained nuclei, Figure $\mathbf{7 B}$ ) and in vitro migration 
("Transwell” assays, Figure 7C) of koGai3 pOS-1 cells. Furthermore, shRNA-induced silencing of Akt1/2 (Figure 7D) blocked Akt-S6K phosphorylations (Figure 7D) and mimicked Gai3 depletion-induced actions, suppressing pOS-1 cell proliferation (Figure 7E) and in vitro migration (Figure 7F). Significantly, re-introducing the Gai3 shRNA lentivirus or the Gai3-expresing construct (Figure 7G) was unable to further influence cell proliferation (Figure 7E) and migration (see quantified results in Figure 7F) in Akt1/2-silenced pOS-1 cells. Moreover, in the Gai3-overexpressed pOS-1 cells (OE-Gai3-sL1), treatment with LY294002, a PI3K-Akt inhibitor [35], blocked Akt-S6K phosphorylation (Figure $7 \mathbf{H}$ ) and inhibited cell proliferation (Figure 7I) and in vitro cell migration (Figure 7J). Thus, Akt-mTOR inhibition should be responsible for Gai3 depletion-induced anti-OS cell activity.

\section{Gai3 depletion inhibits OS cell growth in vivo}

At last, pOS-1 cells were subcutaneously (s.c.) injected to the nude mice. Within 20 days of cell inoculation, the subcutaneous pOS-1 xenograft tumors were established and each tumor volume was close to $100 \mathrm{~mm}^{3}$ ("Day-0"). The xenograft-bearing mice were thereafter assigned into three different groups randomly, with six mice in every group $(\mathrm{n}=$ 6). Afterwards, the mice were intratumorally injected daily with the AAV-packed Gai3 shRNAs (AAV-shGai3-seq1 or AAV-sh-Gai3-seq2, two different sequences in the AAV9 construct) or AAV-packed control shRNA (AAV-shC), for 12 consecutive days. Figure 8A, recording tumor growth, demonstrated that the growth of pOS-1 xenograft tumors was robustly mitigated after AAV-sh-Gai3 injection. The volumes of AAV-sh-Gai3-injected pOS-1 xenografts were dramatically lower than those of AAV-shCinjected pOS-1 xenografts (Figure 8A). The estimated daily tumor growth (see previous studies [22, 23]) results demonstrated that subcutaneous pOS-1 xenograft growth was dramatically suppressed with AAV-sh-Gai3 injection in the nude mice (Figure 8B). At Day-42, all the animals were anaesthetized and decapitated, and pOS-1 xenografts carefully isolated and weighted. The pOS-1 tumors with AAV-sh-Gai3 injection were significantly lighter than pOS-1 tumors with the control shRNA virus injection (Figure 8C). Among the three mice groups there was no significant difference in the mice body weights (Figure 8D).
A.

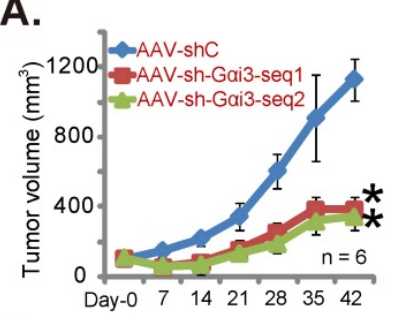

E.

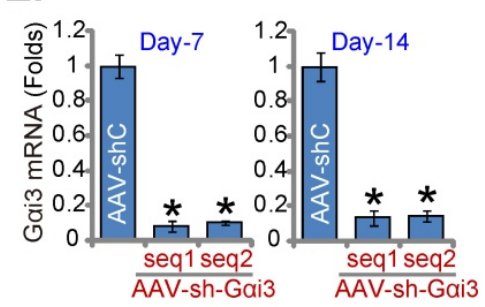

B.
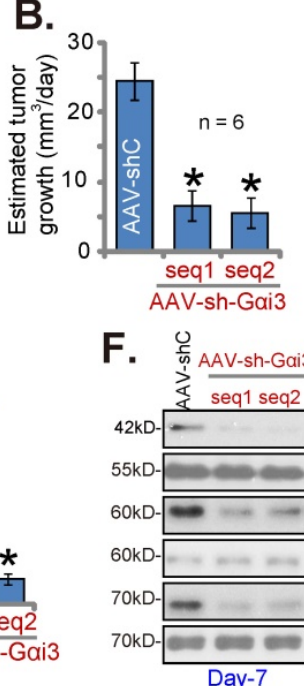

C.

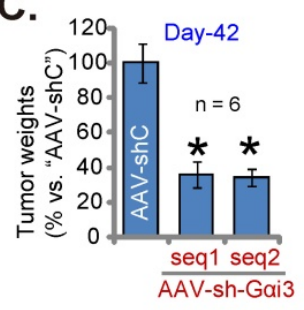

D.
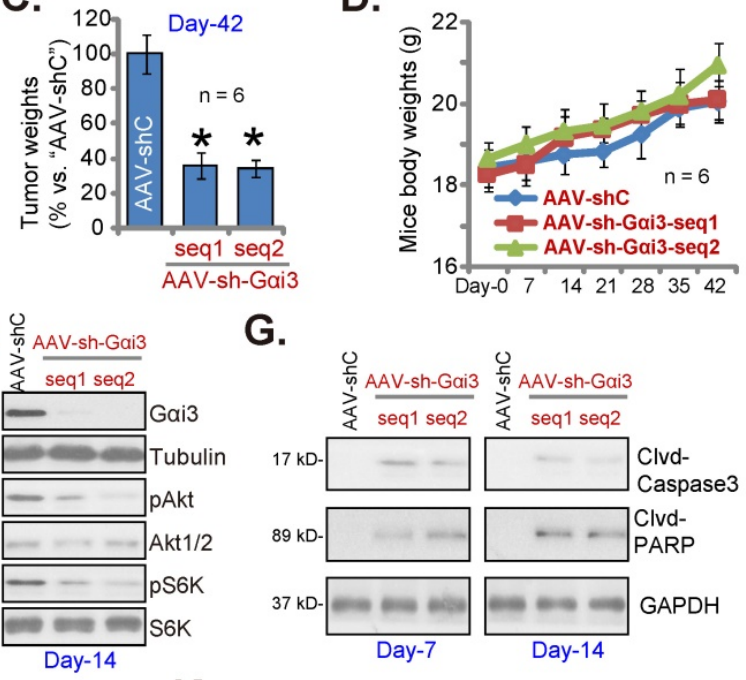

G.

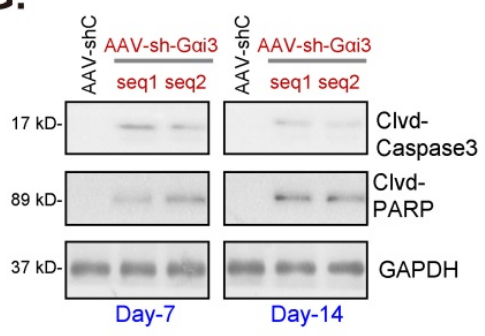

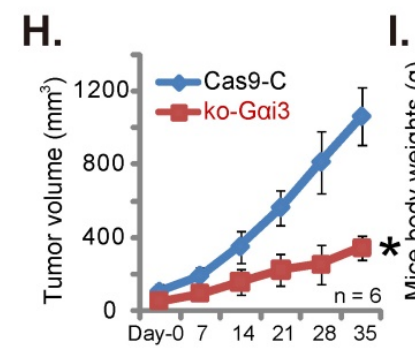

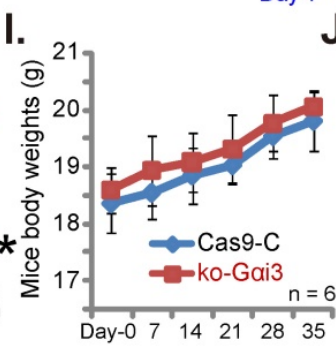

J.

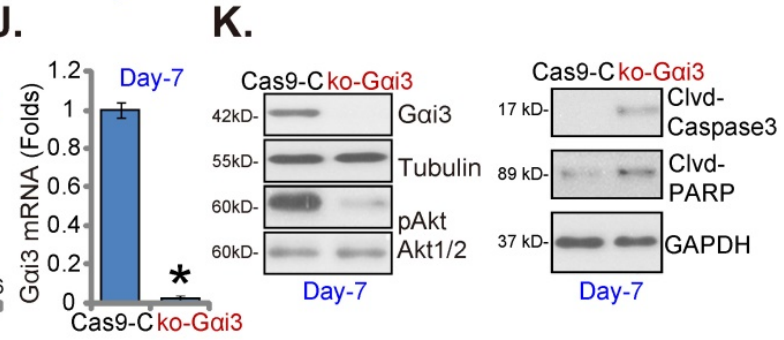

Figure 8. Gai3 depletion inhibits OS cell growth in vivo. The pOS-1 xenografts-bearing nude mice were intratumorally injected (daily) with the AAV-packed Gai3 shRNAs (AAV-sh-Gai3-seql or AAV-sh-Gai3-seq2, two different sequences) or AAV-packed control shRNA (AAV-shC) for 12 days. The mice images was shown (A); The tumor volumes (A) and the mice body weights (D) were recorded weekly ("Day-0" to "Day-42", total 42 days). The estimated daily tumor growth was calculated and presented (B). At Day-42, all pOS-1 xenograft tumors were isolated and weighted (C). Expression of listed genes and proteins in indicated tumor tissues lysates was shown (E-G) assays. The ko-Gai3 pOS-1 cells and Ca9-C control cells were s.c. injected to the nude mice. After 20 days, tumor volumes recordings were started ("Day-0"). Weekly tumor volumes (H) and the mice body weights (I) were presented. Expression of listed genes and proteins in the described tumor lysates was tested (J and $\mathbf{K})$. All blotting data in this Figure were repeated five times. *P $<0.05$ versus "AAV-shC"/“Ca9-C" group. 
At experimental Day-7 and Day-14, 3h after virus injection, one tumor of each group was carefully isolated, and total six tumors were obtained. Goi3 mRNA was dramatically decreased in AAV-sh-Gai3injected pOS-1 xenograft tissues (Figure 8E), where Gai3 protein downregulation as well as p-Akt and p-S6K inhibition were detected (Figure 8F). Supporting apoptosis activation, we showed that cleaved-caspsae-3/cleaved-PARP levels were augmented in Gai3-silenced pOS-1 xenograft tissues (Figure 8G). Thus, intratumoral injection of AAVpacked Gai3 shRNA suppressed Akt-mTOR activation and provoked apoptosis in pOS-1 xenografts.

In addition the ko-Gai3 pOS-1 cells and the Ca9-C control cells (see Figure 4) were injected to the nude mice, forming subcutaneous xenografts. After 20 days, tumor recordings were started ("Day-0"). As shown ko-Gai3 pOS-1 xenograft growth was slower than the Ca9-C xenografts (Figure $8 \mathbf{H}$ ), while animal body weights were indifferent (Figure 8I). At experimental Day-7, we carefully isolated one tumor xenograft per group. Gai3 mRNA and protein (Figure 8J-K) expression was completely depleted in ko-Gai3 pOS-1 xenograft tissues, where p-Akt was decreased (Figure 8K). The cleaved-caspsae-3 and the cleaved-PARP levels were increased in Gai3-KO xenograft tissues (Figure $8 \mathrm{~K}$ ), supporting apoptosis induction in vivo.

\section{Discussion}

The GPCR superfamily is composed of the immense structural and functional different proteins, participating in various biological processes and signals in the bone [36]. Due to gene mutations, depletion or overexpression, GPCR components are dysregulated in human OS [36]. Their roles in OS progression have been established [36]. For example, Iyer et al., found that $\mathrm{A} 3$ adenosine receptor (A3AR) depletion activated protein kinase A (PKA)-Aktnuclear factor (NF)-kB signaling to promote OS cell growth [37]. High GPR56 (G protein-coupled receptor 56) expression is an unfavorable prognostic factor, promoting invasion and proliferation of OS cells [38]. Liu et al. demonstrated that GPR110 (G proteincoupled receptor 110) silencing inhibited OS cell growth [39].

Importantly, studies have reported that Gai-coupled GPCRs, including Apelin receptors [40], CXCR4 [41-43], melatonin receptors [44], are important contributor for OS progression. Due to various gene mutations (or overexpression), concurrent and sustained activation of multiple different RTKs in OS will provoke sustained activation of oncogenic signaling, leading to persistent OS growth and progression $[4,8,9]$. Interestingly, we have previously shown that Gai proteins are essential for signalings by several important oncogenic RTKs (including EGFR [20], VEGFR2 [16], KGFR [19], FGFR [18] and TrkB [17]) as well as the non-RTK receptor (IL-4R [15]).

After showing the essential role of Gai 1/3 in activation of oncogenic signalings by RTKs, we previously explored Gai $1 / 3$ in different human cancers. Gai 1 and Gai 3 expression is elevated in glioma, correlating with tumor stage [14, 18]. Gai1 can form a complex with multiple RTKs (including FGFR, PDGFR and EGFR), transducing downstream Akt-mTOR activation in glioma tissues and cells [18]. Conversely, Gail silencing or mutation inhibited glioma cell growth [18]. In the mouse brain, the orthotopic growth of patient-derived glioma xenografts was arrested after Gai 1/3 depletion, whereas forced overexpression of Gai $1 / 3$ enhanced growth [14]. We also showed that Gai 1 upregulation in human gastric cancer was correlated with poor overall survival [21]. Gai 1 silencing or knockout inhibited Akt-mTOR activation and gastric cancer cell growth [21]. These previous studies supported that Gai $1 / 3$ could be important oncogenic genes and promising therapeutic targets of human cancer.

Gai3 should be a vital gene for OS progression. TCGA database shows that transcripts of Goi3 are significantly upregulated in sarcoma tissues, and high-Gai3 expression in sarcoma correlating with the poor overall survival. Gai3 elevation was observed in local OS tissues as well as in different immortalized and primary OS cells, while low expression was observed in cancer-surrounding normal bone tissues and in immortalized and primary osteoblasts. Functional studies showed that in different OS cells, Gai3 depletion, by shRNA or CRISPR/Cas9 strategies, robustly suppressed cell survival, proliferation and cell migration, and provoking G1-S arrest and apoptosis. Contrarily, ectopic Gai3 overexpression can further accelerate OS cell growth. In vivo, Gai3 shRNA AAV intratumoral injection potently suppressed the growth of the patient-derived OS xenografts in nude mice. Moreover, the growth of primary OS xenografts of the Gai3 KO cells was largely suppressed.

We have previously discovered that Gai1/3 association with multiple RTKs was required for downstream signaling activation. For instance, Gai1/3 are key proteins in mediating VEGF-induced VEGFR2 signaling [16]. Following VEGF stimulation, Gai1/3 were in the VEGFR2 endocytosis complex, required for VEGFR2 endocytosis and subsequent activation of downstream signalings [16]. Similarly, Gai1/3 proteins are indispensable signaling molecule 
for EGF- and KGF-induced Akt-mTORC1 signaling activation [19, 20]. In addition, brain-derived neurotrophic factor (BDNF)-induced signaling and anti-depressive actions required Gai1/3 [17]. Gai 1/3 silencing inhibited BDNF-induced TrkB endocytosis and activation of the downstream signaling [17].

The present study implied that Gai3-driven OS cell growth was primarily through mediating Akt-mTOR cascade activation. In OS cell and tissues Gai3 associated with RTKs (VEGFR2, FGFR, PGDFR and EGFR), essential for downstream Akt-mTOR activation. In OS cells Akt-S6K activation was robustly suppressed by Gai3 shRNA/KO, but augmented after Gai3 overexpression. In vivo, Akt-S6K phosphorylations were decreased in Gai3-depleted OS xenografts tissues. Importantly, disrupting Gai3-RTKs association, through dnGai3, largely inhibited Akt-S6K activation and OS cell proliferation and migration. Restoring Akt-S6K activation, by caAkt1, rescued proliferation and migration of Gai3-KO OS cells. Conversely, mimicking Gai3 depletion-induced actions, Akt1/2 silencing inhibited OS cell proliferation and migration. Significantly, exogenously altering Gai3 expression failed to affect proliferation and migration in Akt1/2-silenced cells. Therefore, Gai3-driven OS cell growth was possibly due to mediating RTKs-Akt signaling.

\section{Conclusion}

Over three-fifths of bone sarcoma are OS $[45,46]$. The standard chemotherapy of OS in clinic is the combination of methotrexate, doxorubicin, and cisplatin [45, 47, 48], showing limited success in metastatic and other OS patients with advanced diseases [45, 47, 48]. Further exploring key pathologic mechanisms and the driving signaling molecule for advanced OS is therefore important [45, 47, 48]. The results of this study showed that overexpressed Gai3 mediated RTKs signaling to drive OS progression, serving as a novel and promising treatment molecular target for patients with OS.

\section{Acknowledgements}

This work was generously supported by grants from the National Natural Science Foundation of China (81922025, 81974388, 81873995, 81974334, 81571282 and 81771457), Development Fund of the Affiliated Hospital of Xuzhou Medical University (XYFM2021043), and Suqian Sci\&Tech Program (K202016), a Project Funded by the Priority Academic Program Development of Jiangsu Higher Education Institutions. The funders had no role in the study design, data collection and analysis, decision to publish, or preparation of the manuscript.

\section{Ethics Statement}

This study was approved by Ethics Committee of Soochow University.

\section{Data Availability Statement}

All data are available upon request.

\section{Author Contributions}

All the listed authors conceived, designed, and supervised the study. All listed authors collected samples, performed the experiments and analyzed the data. All authors reviewed and approved the final manuscript.

\section{Competing Interests}

The authors have declared that no competing interest exists.

\section{References}

1. Anderson ME. Update on Survival in Osteosarcoma. Orthop Clin North Am. 2016; 47: 283-92.

2. Zhao J, Dean DC, Hornicek FJ, Yu X, Duan Z. Emerging next-generation sequencing-based discoveries for targeted osteosarcoma therapy. Cancer Lett. 2020; 474: 158-67.

3. Bishop MW, Janeway KA, Gorlick R. Future directions in the treatment of osteosarcoma. Curr Opin Pediatr. 2016; 28: 26-33.

4. Denduluri SK, Wang Z, Yan Z, Wang J, Wei Q, Mohammed MK, et al. Molecular pathogenesis and therapeutic strategies of human osteosarcoma. J Biomed Res. 2015; 30.

5. Liang X, Wang X, He Y, Wu Y, Zhong L, Liu W, et al. Acetylation dependent functions of Rab22a-NeoF1 Fusion Protein in Osteosarcoma. Theranostics. 2020; 10: 7747-57.

6. $\mathrm{Hu}$ XK, Rao SS, Tan YJ, Yin $\mathrm{H}$, Luo MJ, Wang ZX, et al. Fructose-coated Angstrom silver inhibits osteosarcoma growth and metastasis via promoting ROS-dependent apoptosis through the alteration of glucose metabolism by inhibiting PDK. Theranostics. 2020; 10: 7710-29.

7. Wang S, Zhong L, Li Y, Xiao D, Zhang R, Liao D, et al. Up-regulation of PCOLCE by TWIST1 promotes metastasis in Osteosarcoma. Theranostics. 2019; 9: 4342-53.

8. Tian Z, Niu X, Yao W. Receptor Tyrosine Kinases in Osteosarcoma Treatment: Which Is the Key Target? Front Oncol. 2020; 10: 1642.

9. Rettew AN, Getty PJ, Greenfield EM. Receptor tyrosine kinases in osteosarcoma: not just the usual suspects. Adv Exp Med Biol. 2014; 804: 47-66.

10. Fan H, Li P, Zingarelli B, Borg K, Halushka PV, Birnbaumer L, et al. Heterotrimeric Galpha(i) proteins are regulated by lipopolysaccharide and are anti-inflammatory in endotoxemia and polymicrobial sepsis. Biochim Biophys Acta. 2011; 1813: 466-72.

11. Broxmeyer HE, Youn BS, Kim C, Hangoc G, Cooper S, Mantel C. Chemokine regulation of hematopoiesis and the involvement of pertussis toxin-sensitive $G$ alpha i proteins. Ann N Y Acad Sci. 2001; 938: 117-27; discussion 27-8.

12. Pines M, Santora A, Gierschik P, Menczel J, Spiegel A. The inhibitory guanine nucleotide regulatory protein modulates agonist-stimulated cAMP production in rat osteosarcoma cells. Bone Miner. 1986; 1: 15-26.

13. Wang JW, Su W, Law YP, Lu CH, Chen YC, Wang JL, et al. Mechanism of bradykinin-induced $\mathrm{Ca}(2+)$ mobilization in MG63 human osteosarcoma cells. Horm Res. 2001; 55: 265-70.

14. Wang $Y$, Liu YY, Chen MB, Cheng KW, Qi LN, Zhang ZQ et al. Neuronal-driven glioma growth requires Galphai1 and Galphai3. Theranostics. 2021; 11: 8535-49.

15. Bai JY, Li Y, Xue GH, Li KR, Zheng YF, Zhang ZQ et al. Requirement of Galphai1 and Galphai3 in interleukin-4-induced signaling, macrophage M2 polarization and allergic asthma response. Theranostics. 2021; 11: 4894-909.

16. Sun J, Huang W, Yang SF, Zhang XP, Yu Q, Zhang ZQ, et al. Galphai1 and Galphai3mediate VEGF-induced VEGFR2 endocytosis, signaling and angiogenesis. Theranostics. 2018; 8: 4695-709.

17. Marshall J, Zhou XZ, Chen G, Yang SQ, Li Y, Wang Y, et al. Antidepression action of BDNF requires and is mimicked by Galphai1/3 expression in the hippocampus. Proc Natl Acad Sci U S A. 2018; 115: E3549-E58.

18. Liu YY, Chen MB, Cheng L, Zhang ZQ, Yu ZQ, Jiang Q, et al. microRNA-200a downregulation in human glioma leads to Galphai1 over-expression, Akt activation, and cell proliferation. Oncogene. 2018; 37: 2890-902.

19. Zhang YM, Zhang ZQ, Liu YY, Zhou X, Shi XH, Jiang Q, et al. Requirement of Galphai1/3-Gab1 signaling complex for keratinocyte growth factor-induced PI3K-AKT-mTORC1 activation. J Invest Dermatol. 2015; 135: 181-91. 
20. Cao C, Huang X, Han Y, Wan Y, Birnbaumer L, Feng GS, et al. Galpha(i1) and Galpha(i3) are required for epidermal growth factor-mediated activation of the Akt-mTORC1 pathway. Sci Signal. 2009; 2: ra17.

21. Lv Y, Wang Y, Song Y, Wang SS, Cheng KW, Zhang ZQ, et al. LncRNA PINK1-AS promotes $G$ alpha i1-driven gastric cancer tumorigenesis by sponging microRNA-200a. Oncogene. 2021; 40: 3826-44.

22. Shan HJ, Zhu LQ, Yao C, Zhang ZQ, Liu YY, Jiang Q, et al. MAFG-driven osteosarcoma cell progression is inhibited by a novel miRNA miR-4660. Mol Ther Nucleic Acids. 2021; 24: 385-402.

23. Gao YY, Ling ZY, Zhu YR, Shi C, Wang Y, Zhang XY, et al. The histone acetyltransferase $\mathrm{HBO} 1$ functions as a novel oncogenic gene in osteosarcoma. Theranostics. 2021; 11: 4599-615.

24. Guo S, Chen C, Ji F, Mao L, Xie Y. PP2A catalytic subunit silence by microRNA-429 activates AMPK and protects osteoblastic cells from dexamethasone. Biochem Biophys Res Commun. 2017; 487: 660-5.

25. Zhu CY, Yao C, Zhu LQ, She C, Zhou XZ. Dexamethasone-induced cytotoxicity in human osteoblasts is associated with circular RNA HIPK3 downregulation. Biochem Biophys Res Commun. 2019; 516: 645-52.

26. Fan JB, Liu W, Zhu XH, Cui SY, Cui ZM, Zhao JN. microRNA-7 inhibition protects human osteoblasts from dexamethasone via activation of epidermal growth factor receptor signaling. Mol Cell Biochem. 2019; 460: 113-21.

27. Zhang D, Xia H, Zhang W, Fang B. The anti-ovarian cancer activity by WYE-132, a mTORC1/2 dual inhibitor. Tumour Biol. 2016; 37: 1327-36.

28. Yang H, Zhao J, Zhao M, Zhao L, Zhou LN, Duan Y, et al. GDC-0349 inhibits non-small cell lung cancer cell growth. Cell Death Dis. 2020; 11: 951.

29. Lv Y, Si M, Chen N, Li Y, Ma X, Yang H, et al. TBX2 over-expression promotes nasopharyngeal cancer cell proliferation and invasion. Oncotarget. 2017; 8: 52699-707.

30. Shao NY, Wang DX, Wang Y, Li Y, Zhang ZQ, Jiang Q, et al. MicroRNA-29a-3p Downregulation Causes Gab1 Upregulation to Promote Glioma Cell Proliferation. Cell Physiol Biochem. 2018; 48: 450-60.

31. Zhang X, Shan H, Zhang P, C S, XZ Z. LncRNA EPIC1 protects human osteoblasts from dexamethasone-induced cell death. Biochemical and biophysical research communications. 2018; 503: 2255-62.

32. Chen MB, Liu $Y Y$, Xing ZY, Zhang ZQ, Jiang $Q, L u ~ P H$, et al. Itraconazole-Induced Inhibition on Human Esophageal Cancer Cell Growth Requires AMPK Activation. Mol Cancer Ther. 2018; 17: 1229-39.

33. Wang SS, Lv Y, Xu XC, Zuo Y, Song Y, Wu GP, et al. Triptonide inhibits human nasopharyngeal carcinoma cell growth via disrupting Lnc-RNA THOR-IGF2BP1 signaling. Cancer Lett. 2019; 443: 13-24.

34. Zuo Y, Wang Y, Hu H, Cui W. Atorvastatin Protects Myocardium Against Ischemia-Reperfusion Injury Through Inhibiting miR-199a-5p. Cell Physiol Biochem. 2016; 39: 1021-30.

35. Brunn GJ, Williams J, Sabers C, Wiederrecht G, Lawrence JC, Jr., Abraham RT. Direct inhibition of the signaling functions of the mammalian target of rapamycin by the phosphoinositide 3-kinase inhibitors, wortmannin and LY294002. EMBO J. 1996; 15: 5256-67.

36. Luo J, Sun P, Siwko S, Liu M, Xiao J. The role of GPCRs in bone diseases and dysfunctions. Bone Res. 2019; 7: 19.

37. Iyer SV, Ranjan A, Elias HK, Parrales A, Sasaki H, Roy BC, et al. Genome-wide RNAi screening identifies TMIGD3 isoform1 as a suppressor of NF-kappaB and osteosarcoma progression. Nat Commun. 2016; 7: 13561.

38. Chen Z, Gao P, Li Z. Expression of G Protein-coupled Receptor 56 Is an Unfavorable Prognostic Factor in Osteosarcoma Patients. Tohoku J Exp Med. 2016; 239: 203-11.

39. Liu Z, Zhang G, Zhao C, Li J. Clinical Significance of G Protein-Coupled Receptor 110 (GPR110) as a Novel Prognostic Biomarker in Osteosarcoma. Med Sci Monit. 2018; 24: 5216-24.

40. Cui L, Zhang JY, Ren ZP, Zhao HJ, Li GS. APLNR promotes the progression of osteosarcoma by stimulating cell proliferation and invasion. Anticancer Drugs. 2019; 30: 940-7.

41. Guan G, Zhang Y, Lu Y, Liu L, Shi D, Wen Y, et al. The HIF-1alpha/CXCR4 pathway supports hypoxia-induced metastasis of human osteosarcoma cells. Cancer Lett. 2015; 357: 254-64.

42. Laverdiere $\mathrm{C}$, Hoang $\mathrm{BH}$, Yang $\mathrm{R}$, Sowers $\mathrm{R}$, Qin $\mathrm{J}$, Meyers PA, et al. Messenger RNA expression levels of CXCR4 correlate with metastatic behavior and outcome in patients with osteosarcoma. Clin Cancer Res. 2005; 11: 2561-7.

43. Zhang P, Dong L, Yan K, Long H, Yang TT, Dong MQ, et al. CXCR4-mediated osteosarcoma growth and pulmonary metastasis is promoted by mesenchymal stem cells through VEGF. Oncol Rep. 2013; 30: 1753-61.

44. Lu KH, Lin RC, Yang JS, Yang WE, Reiter RJ, Yang SF. Molecular and Cellular Mechanisms of Melatonin in Osteosarcoma. Cells. 2019; 8.

45. Isakoff M, Bielack S, Meltzer P, R G. Osteosarcoma: Current Treatment and a Collaborative Pathway to Success. Journal of clinical oncology : official journal of the American Society of Clinical Oncology. 2015; 33: 3029-35.

46. Lin Y, Jewell B, Gingold J, L L, R Z, LL W, et al. Osteosarcoma: Molecular Pathogenesis and iPSC Modeling. Trends in molecular medicine. 2017; 23: 737-55.

47. Luetke A, Meyers P, Lewis I, H J. Osteosarcoma treatment - where do we stand? A state of the art review. Cancer treatment reviews. 2014; 40: 523-32.

48. Zhang $\mathrm{Y}$, Yang J, Zhao N, C W, S K, Y Z, et al. Progress in the chemotherapeutic treatment of osteosarcoma. Oncology letters. 2018; 16: 6228-37. 